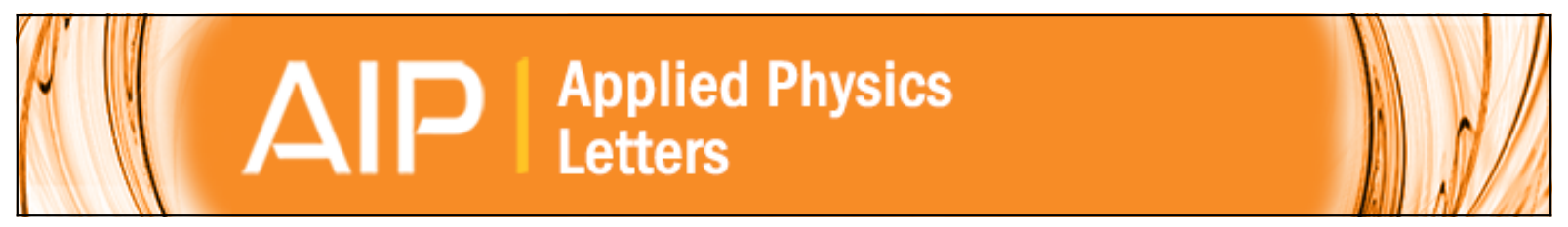

\title{
Heteroepitaxal fabrication and structural characterizations of ultrafine GaN/ZnO coaxial nanorod heterostructures
}

Sung Jin An, Won II Park, Gyu-Chul Yi, Yong-Jin Kim, Hee-Bok Kang, and Miyoung Kim

Citation: Applied Physics Letters 84, 3612 (2004); doi: 10.1063/1.1738180

View online: http://dx.doi.org/10.1063/1.1738180

View Table of Contents: http://scitation.aip.org/content/aip/journal/apl/84/18?ver=pdfcov

Published by the AIP Publishing

\section{Articles you may be interested in}

Structural and resistance switching properties of epitaxial $\mathrm{Pt} / \mathrm{ZnO} / \mathrm{TiN} / \mathrm{Si}(001)$ heterostructures

J. Appl. Phys. 115, 234501 (2014); 10.1063/1.4883959

Microstructural compositional, and optical characterization of GaN grown by metal organic vapor phase epitaxy on $\mathrm{ZnO}$ epilayers

J. Vac. Sci. Technol. B 27, 1655 (2009); 10.1116/1.3137967

Strain relaxation in AIGaN multilayer structures by inclined dislocations

J. Appl. Phys. 105, 083507 (2009); 10.1063/1.3087515

Nanostructural characterization and two-dimensional electron-gas properties in high-mobility Al Ga N/Al N/Ga $\mathrm{N}$ heterostructures grown on epitaxial AIN/sapphire templates

J. Appl. Phys. 98, 063713 (2005); 10.1063/1.2060946

Structural characterization of strained AIGaN layers in different Al content Al Ga N/Ga N heterostructures and its effect on two-dimensional electron transport propertiesa)

J. Vac. Sci. Technol. B 23, 1527 (2005); 10.1116/1.1993619

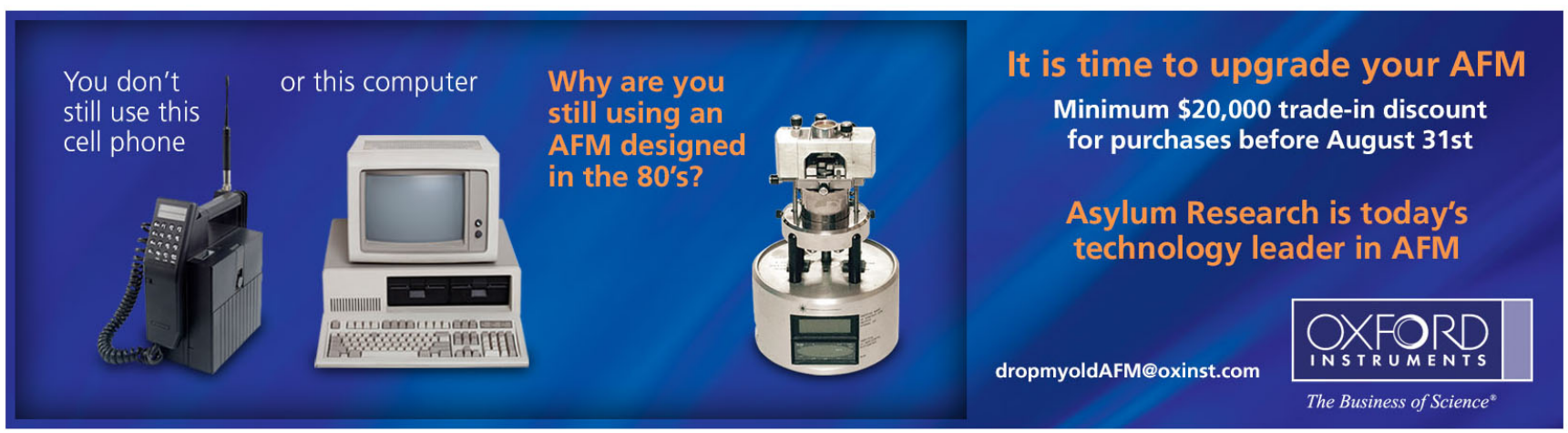




\title{
Heteroepitaxal fabrication and structural characterizations of ultrafine GaN/ZnO coaxial nanorod heterostructures
}

\author{
Sung Jin An, Won II Park, and Gyu-Chul Yja) \\ Department of Materials Science and Engineering, Pohang University of Science and Technology \\ (POSTECH), Pohang 790-784, Korea \\ Yong-Jin Kim and Hee-Bok Kang \\ LG Siltron Advanced Materials Research Center, 283, Imsoo-dong, Gumi, Kyoungbuk 730-724, Korea \\ Miyoung Kim \\ Samsung Advanced Institute of Technology, P.O. Box 111, Suwon 440-600, Korea
}

(Received 16 February 2004; accepted 10 March 2004; published online 20 April 2004)

\begin{abstract}
We report on heteroepitaxial fabrication and structural characterizations of ultrafine GaN/ZnO coaxial nanorod heterostructures. The coaxial nanorod heterostructures were fabricated by epitaxial growth of a GaN layer on ultrafine $\mathrm{ZnO}$ nanorods. Epitaxial growth and precise control of GaN overlayer thickness were obtained by low pressure metalorganic vapor-phase epitaxy. $\mathrm{ZnO}$ nanorods grown on $\mathrm{Si}$ and sapphire substrates using catalyst-free metalorganic chemical vapor deposition exhibited diameters as small as $7 \mathrm{~nm}$. Furthermore, structural properties of the coaxial nanorod heterostructures were investigated using both synchrotron-radiation x-ray diffraction and high resolution transmission electron microscopy. (C) 2004 American Institute of Physics.
\end{abstract}

[DOI: $10.1063 / 1.1738180]$

Composition-modulated semiconductor nanorod heterostructures greatly increase the versatility and power of nanometer-scale electronics and optoelectronic building blocks. ${ }^{1-3}$ Recent fabrication of semiconductor coaxial nanorod heterostructures showing composition modulation along the nanorod radial direction is of particular high interest. ${ }^{4-7}$ Although coaxial structures exhibiting a thick diameter of 20-100 nm have previously been applied to nano-scale high electron mobility transistors (HEMTs), ${ }^{5}$ the device performance may be enhanced by using ultrafine coaxial heterostructures due to one-dimensional (1D) quantum confinement effects in core nanorods. Additionally, a well-defined, clean interface in the heterostructures is very important for excellent device performance and reliability since defects at the interface deteriorate device characteristics. For 1D coaxial heterostructures, in particular, the effect of interfacial defects on material properties becomes more significant due to their high interface to volume ratio. Nevertheless, interfacial defects in 1D nanorod heterostructures have rarely been investigated to date. Here we report on the synthesis of ultrafine $\mathrm{GaN} / \mathrm{ZnO}$ coaxial nanorod heterostructures by heteroepitaxy and their structural defect characterizations.

$\mathrm{GaN} / \mathrm{ZnO}$ coaxial nanorod heterostructures were fabricated on $\mathrm{Al}_{2} \mathrm{O}_{3}$ and $\mathrm{Si}$ substrates using catalyst-free metalorganic vapor phase epitaxy (MOVPE). ZnO nanorods were prepared as a core nanomaterial using diethylzinc and oxygen as the reactants. ${ }^{4,8}$ After the $\mathrm{ZnO}$ nanorod preparation, $\mathrm{GaN}$ layers were epitaxially grown on the $\mathrm{ZnO}$ nanorods using trimethyl-Ga (TMGa) and ammonia in another MOVPE chamber. Typical flow rates of TMGa and ammonia were 3.0 and 500-1000 sccm, respectively. Growth tempera-

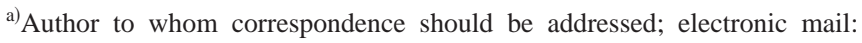
gcyi@ postech.ac.kr
}

ture was in the range of $400-700^{\circ} \mathrm{C}$. The mean diameters of $\mathrm{GaN} / \mathrm{ZnO}$ coaxial nanorod heterostructures were about 14 , 20 , and $32 \mathrm{~nm}$ for 1,5 , and $10 \mathrm{~min}$ growth, respectively. Since the mean diameter of ultrafine $\mathrm{ZnO}$ core nanorods is $7-8 \mathrm{~nm}$, the GaN layer thickness is $2-3$ and $5-7 \mathrm{~nm}$ for 1 and $5 \mathrm{~min}$ growth, respectively, as also confirmed using high resolution transmission electron microscopy (HRTEM). ${ }^{4,9}$

Synchrotron radiation $\mathrm{x}$-ray diffraction (SR-XRD) was employed for the structural characterization of coaxial nanorod heterostructures. The SR-XRD measurements of GaN/ $\mathrm{ZnO}$ nanorod heterostructures were performed at the 1.54019 $\AA$ wavelength using a four-circle diffractometer of the $3 \mathrm{C} 2$ SR-XRD beam line at the Pohang Accelerator Laboratory (PAL). ${ }^{9}$

Figure 1 shows the general morphology of GaN/ZnO

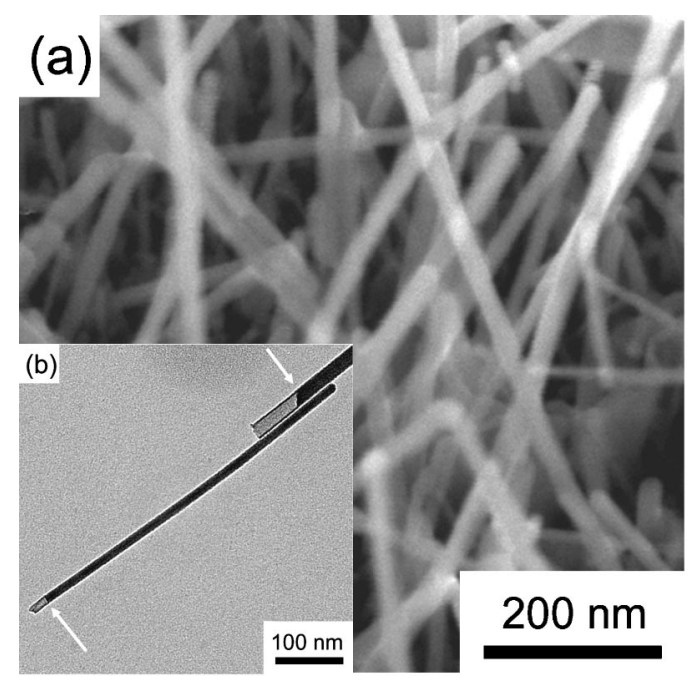

FIG. 1. FESEM image and low magnification TEM image of GaN/ZnO coaxial nanorod heterostructures (inset). 


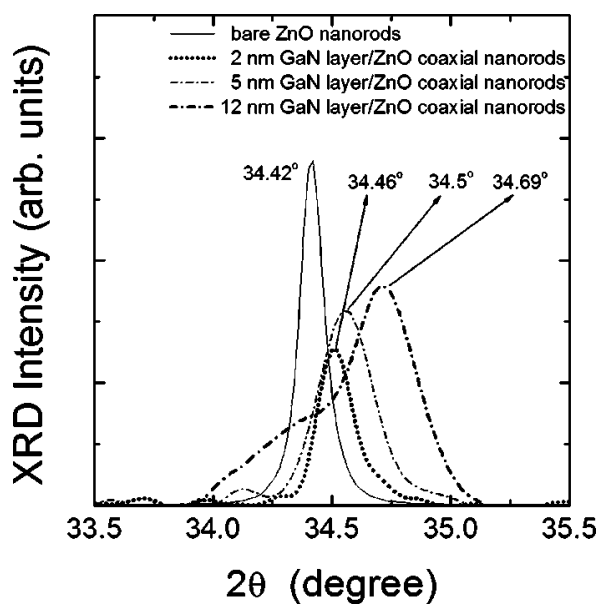

FIG. 2. SR-XRD $\theta-2 \theta$ scan of bare $\mathrm{ZnO}$ nanorods and $\mathrm{GaN} / \mathrm{ZnO}$ coaxial nanorod heterostructures with various $\mathrm{GaN}$ thicknesses of 2,5 , and $12 \mathrm{~nm}$.

nanorod heterostructures investigated using field-emission scanning electron microscopy (FESEM). Although the vertical alignment of coaxial nanorod heterostructures was not as good as that of thick $\mathrm{ZnO}$ nanorods, the nanorod density was $1.6 \times 10^{10} / \mathrm{cm}^{2}$. Further structural characterization of GaN/ $\mathrm{ZnO}$ coaxial nanorod heterostructures was investigated using TEM. As shown in the inset, the low magnification TEM image shows that the $\mathrm{GaN}$ layer extends all along the $\mathrm{ZnO}$ nanorod with different brightness at the core and shell layers, indicating that the GaN layers were uniformly coated on $\mathrm{ZnO}$ core nanorods. The coating was robust and $\mathrm{GaN}$ layers were not easily peeled off from the $\mathrm{ZnO}$ core nanorods. As indicated by the arrow in the inset, however, some TEM images show $\mathrm{GaN} / \mathrm{ZnO}$ coaxial nanorod heterostructure and $\mathrm{GaN}$ nanotubes formed during fabrication of the nanorod heterostructures or removal of $\mathrm{GaN} / \mathrm{ZnO}$ coaxial nanorod heterostructures from the substrate. ${ }^{7}$

Figure 2 shows SR-XRD results of GaN/ZnO ultrafine nanorod heterostructures with various $\mathrm{GaN}$ thicknesses of 0 , 2, 5, and $12 \mathrm{~nm}$. The diameters of the core $\mathrm{ZnO}$ nanorods were in the range of $6-10 \mathrm{~nm}$. As shown in Fig. 2, homogeneous core $\mathrm{ZnO}$ nanorods showed a dominant diffraction peak centered at $34.42^{\circ}$ corresponding to the $\mathrm{ZnO}(0002)$ plane. However, SR-XRD data of the GaN/ZnO coaxial nanorod heterostructures exhibited a slight increase in peak position from $34.42^{\circ}$ to $34.69^{\circ}$ depending on the $\mathrm{GaN}$ layer thickness coated on $\mathrm{ZnO}$ nanorods. For $\mathrm{GaN} / \mathrm{ZnO}$ coaxial nanorod heterostructures with GaN layer thicknesses of 2-3 and 5-7 nm, SR-XRD peaks were observed at $34.46^{\circ}$ and $34.5^{\circ}$, respectively. Moreover, the full width at half maximum (FWHM) value in XRD rocking curve data increased with the GaN layer thickness.

The peak position and FWHM depending on the GaN layer thickness presumably results from lattice distortion along the $c$ axis of $\mathrm{GaN}$ due to biaxial in-plane strain. For nanorod heterostructures with a thicker GaN layer, more strain is induced, hence increasing both XRD peak shift and FWHM values. Since the $a$ - and $c$-axis lattice constants are 3.186 and $5.178 \AA$ for $\mathrm{GaN}$ and 3.249 and $5.205 \AA$ for $\mathrm{ZnO}$, respectively, epitaxial $\mathrm{GaN}$ layers and $\mathrm{ZnO}$ nanorods in the heterostructures must have tensile and compressive strain, respectively. ${ }^{10}$ Since the thickness of GaN is relatively thin-
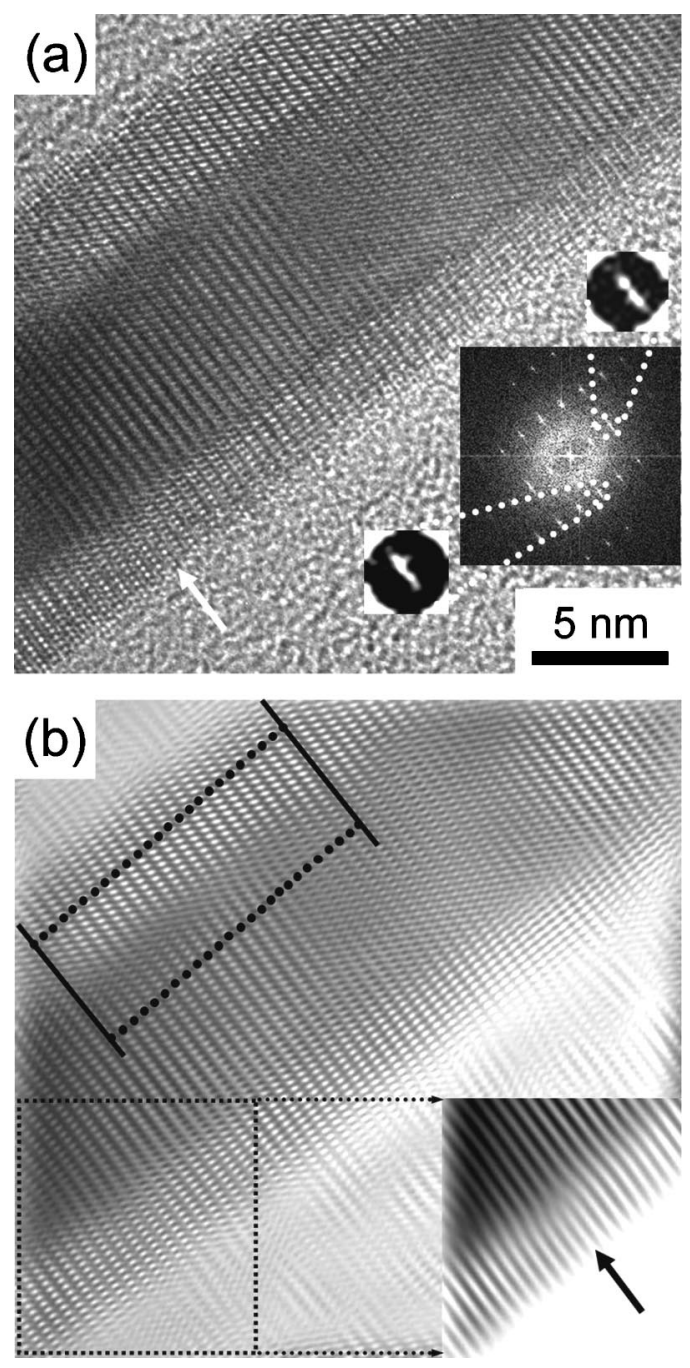

FIG. 3. (a) HRTEM image of a GaN/ZnO coaxial nanorod heterostructure and (b) its filtered and inverse FFT image (inset). The inset in (a) shows an electron diffraction pattern of the $\mathrm{GaN} / \mathrm{ZnO}$ coaxial nanorod heterostructure. The peak splitting indicates the slight difference between $\mathrm{GaN}$ and $\mathrm{ZnO}$ lattice constants. As indicated in the inverse FFT image, the lattice of the $\mathrm{GaN}$ layer does not exactly match that of the $\mathrm{ZnO}$ layer, due to the formation of dislocation.

ner than the diameter of $\mathrm{ZnO}$ core nanorods, SR-XRD data show the dominant peak from $\mathrm{ZnO}$ with a slight peak shift due to the compressive strain. As shown in Fig. 2, the peak shift increases with increasing GaN thickness. For SR-XRD data of the nanorod heterostructures with a GaN layer thickness of $12 \mathrm{~nm}$, however, a shoulder at $34.42^{\circ}$, in addition to the dominant peak at $34.69^{\circ}$, is also shown. Lattice constants calculated from XRD peaks are $5.205 \AA$ for $\mathrm{ZnO}$ and 5.178 $\AA$ for $\mathrm{GaN}$, which are in excellent agreement with the bulk values. ${ }^{10}$ This separation in the XRD peak for nanorod heterostructures with a thicker GaN layer is presumably due to strain release. With the continuous growth of $\mathrm{GaN}$ on the $\mathrm{ZnO}$ nanorod core, $\mathrm{GaN}$ layers readjust to bulk $\mathrm{GaN}$ parameters and induced lattice dislocations (semicoherency) at the interface. This may allow the relief of some of the accumulated strain.

Further structural characterization of $\mathrm{GaN} / \mathrm{ZnO}$ coaxial nanorod heterostructures was investigated using HRTEM. As shown in Fig. 3(a), the HRTEM image of a GaN/ZnO coaxial nanorod heterostructure shows an abrupt and semicoherent 

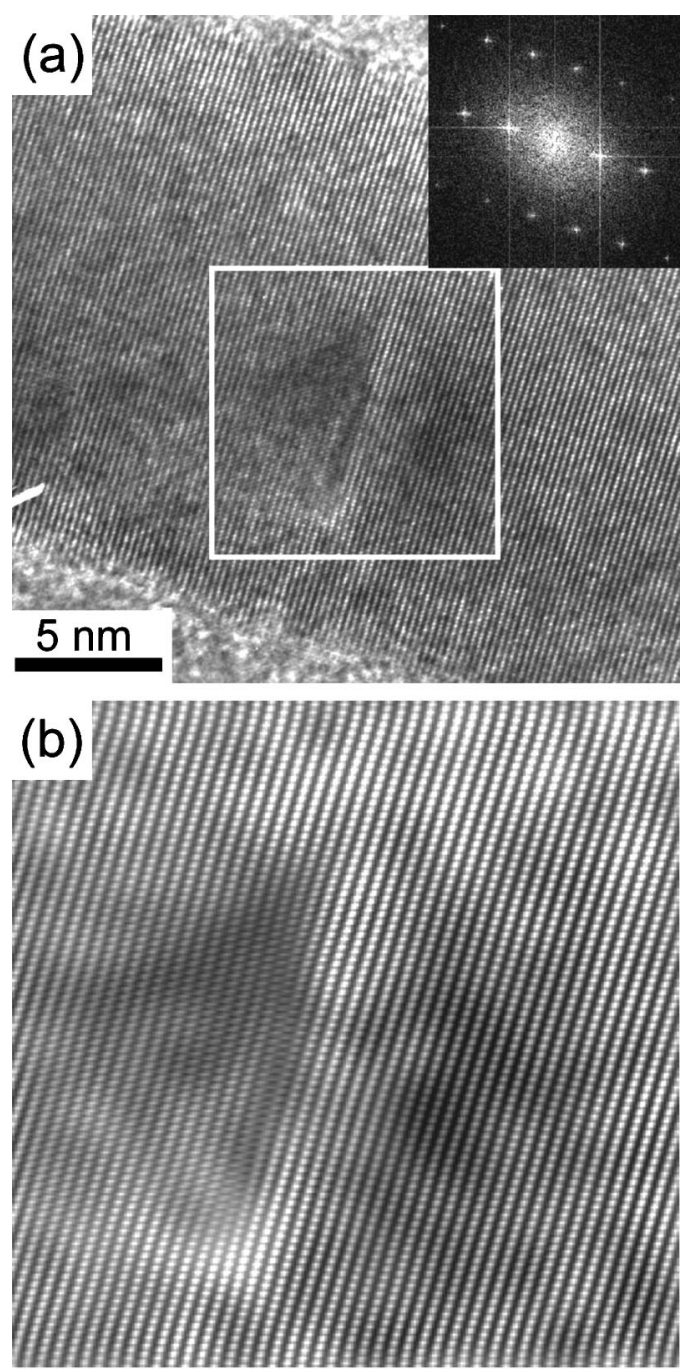

FIG. 4. (a) TEM image and (b) diffractogram of coaxial nanorod heterostructures with stacking faults. The diffractogram was obtained from the white square.

interface between $\mathrm{ZnO}$ and $\mathrm{GaN}$ layers, verifying that the $\mathrm{GaN}$ layers are epitaxially grown on $\mathrm{ZnO}$ nanorods. The inset shows an electron diffraction pattern of a GaN/ZnO coaxial nanorod heterostructure obtained by fast Fourier transform (FFT). The pattern can be indexed as a [100] zone axis hexagonal wurtzite $\mathrm{GaN}$ and $\mathrm{ZnO}$, where two diffraction spots labeled in the inset correspond to planes (0002) and $(01 \overline{1} 0)$. Two peaks corresponding to the $(01 \overline{1} 0)$ reflections of $\mathrm{ZnO}$ and $\mathrm{GaN}$ are distinguished due to the slight difference in lattice constants along the radial direction, indicating radial relaxation of $\mathrm{GaN}$, while the peak splitting of (0002) reflection along the growth direction is not observed, indicating existence of strain from epitaxial growth.

In general, the strain between heteroepitaxial layers induces formation of defects such as misfit dislocations and stacking faults to release the strain. The SR-XRD results show the presence of strain in the nanorod heterostructures.
Hence we further investigate defects near the interface between $\mathrm{ZnO}$ and GaN layers using HRTEM. Despite the observation of the abrupt interface, close examination of the $\mathrm{GaN}$ and $\mathrm{ZnO}$ lattice fringes reveals the presence of edge dislocations as indicated by the arrows in Fig. 3(a). This is shown clearly in the inverse-FFT image in Fig. 3(b), which indicates the formation of the edge dislocation with a Burgers vector in the nanorod growth direction [001]. This type of edge dislocation is rarely observed in pure $\mathrm{ZnO}$ nanorods, but the occurrence is increased in $\mathrm{GaN} / \mathrm{ZnO}$ coaxial nanorod heterostructures, probably due to the lattice mismatch between both structures or the inherent nature of $\mathrm{GaN}$ nanomaterial.

Planar stacking faults are also often observed in coaxial structures. Figures 4(a) and 4(b) show the image and its diffractogram. The diffractogram obtained from the small white square shows streaks along the [001], indicating the existence of stacking faults. The density of these defects, which has not been observed in pure $\mathrm{ZnO}$ nanorods, is about a few tens per micrometer. These results indicate that $\mathrm{GaN}$ was epitaxially grown on $\mathrm{ZnO}$ nanorods, which presumably results from the little lattice mismatch between $\mathrm{GaN}$ and $\mathrm{ZnO}$.

In conclusion, ultrafine $\mathrm{GaN} / \mathrm{ZnO}$ coaxial nanorod heterostructures were fabricated by heteroepitaxial growth of $\mathrm{GaN}$ on $\mathrm{ZnO}$ nanorods. The fabrication of ultrafine coaxial nanorod heterostructures opens up significant opportunities for the fabrication of 1D quantum structure devices including HEMTs. Furthermore, the structural and defect characterization methods used in nanorod heterostructures may readily be expanded to characterize structural defects in many other heteroepitaxial nanomaterials.

This work was supported by LG Siltron Co., the National R\&D Project for Nano Science and Technology, the AEBRC (Grant No. R11-2003-006), the Brain Korea 21 project, and the POSRIP-2003.

${ }^{1}$ W. I. Park, S. W. Jung, Y. H. Jun, and G.-C. Yi, in Proceedings of the 26th International Conference on the Physics of Semiconductors, Edinburgh, UK, 2002, p. 176.

${ }^{2}$ M. S. Gudiksen, L. J. Lauhon, J. Wang, D. C. Smith, and C. M. Lieber, Nature (London) 415, 617 (2002).

${ }^{3}$ W. I. Park, G.-C. Yi, M. Kim, and S. J. Pennycook, Adv. Mater. (Weinheim, Ger.) 15, 526 (2003).

${ }^{4}$ Y. Zhang, K. Suenaga, C. Colliex, and S. Iijima, Science 281, 973 (1998).

${ }^{5}$ L. J. Lauhon, M. S. Gudiksen, D. Wang, and C. M. Lieber, Nature (London) 420, 57 (2002).

${ }^{6}$ H.-M. Lin, Y.-L. Chen, J. Yang, Y.-C. Liu, K.-M. Yin, J.-J. Kai, F.-R. Chen, L.-C. Chen, Y.-F. Chen, and C.-C. Chen, Nano Lett. 3, 537 (2003).

${ }^{7}$ J. Goldberger, R. He, Y. Zhang, S. K. Lee, H. Yan, H.-J. Choi, and P. Yang, Nature (London) 422, 599 (2003).

${ }^{8}$ W. I. Park, D. H. Kim, S.-W. Jung, and G.-C. Yi, Appl. Phys. Lett. 80, 4232 (2002).

${ }^{9}$ S. W. Jung, W. I. Park, G.-C. Yi, and M. Kim, Adv. Mater. (Weinheim, Ger.) 15, 1358 (2003)

${ }^{10}$ F. Hamdani, M. Yeadon, D. J. Smith, H. Tang, W. Kim, A. Salvador, A. E. Botchkarev, J. M. Gibson, A. Y. Polyakov, M. Skowronski, and H. Morkoç, J. Appl. Phys. 83, 983 (1998). 